\title{
Radiological and Clinical Effects of Operative Treatment on Calcaneus Fractures
}

\author{
Khalid Hasan $^{1 *}$, Shamsi Abdul Hameed ${ }^{2}$, Yasir Jameel Ahmed ${ }^{2}$ and Abdul Moeen Baco ${ }^{2}$ \\ ${ }^{1}$ Department of Orthopedic Surgery, Virginia Commonwealth University, Richmond, VA, USA \\ ${ }^{2}$ Department of Orthopedic Surgery, Hamad General Hospital, Doha, Qatar
}

*Corresponding author: Khalid Hasan, Department of Orthopedic Surgery, Virginia

Commonwealth University, Richmond, VA, USA.

Received Date: March 17, 2021

Published Date: March 24, 2021

\begin{abstract}
Background: Study was conducted to determine whether operative treatment for intraarticular calcaneus fractures results in improvement of post-operative Bohler's angle (BA) and if the pre and postoperative measurements of BA can be used as prognostic factor for clinical outcome.

Methods: This retrospective study was conducted at orthopedic department in Hamad General Hospital, Doha, Qatar. The fractures were classified according to BA (Class A: $>16^{\circ}$; Class B: $0-15^{\circ}$; Class $\mathrm{C}:<0^{\circ}$ ). The pre and immediate postoperative radiographs were assessed. Minimum follow up period for assessing clinical outcome was restricted to one year. Patients were telephonically followed up and assessed using the Foot Function Index and American Orthopedic Foot and Ankle Society Ankle Hind Foot Score by two independent observers.

Result and Discussion: 131 intra-articular calcaneus fractures were operated from 117 patients during the study period. Of these, 100 fractures from 96 patients were followed up. Kappa value for inter-observer reliability was 0.6258 (preoperative) and 0.6478 (postoperative). The Cohen kappa coefficient for intra- observer reproducibility was 0.6141 (preoperative) versus 0.6672 (postoperative) measurement of BA. Quantitative analysis was done using the paired t-test. The mean improvement in BA was found to be 13.14 for class A, 20.24 for class B, and 44.75 in class C. The mean AOFAS score for class A, B and C was found to be 46.38, 40.98, and 26.23 respectively. Likewise, the mean FFI was 25.66, 31.42, and 57.63 for class A, B and C respectively.

Conclusion: There was statistically significant improvement in BA among all classes. We conclude that surgical fixation is indicated if the preliminary angle is $0-25^{\circ}$ and it results in good radiologic and functional outcome and grading system of BA can be predictably used as a prognostic indicator.
\end{abstract}

Keywords: Calcaneus; Fractures; Bohler's angle; Operative; Non-operative; functional; Outcome

\section{Introduction}

Calcaneus is the most commonly fractured tarsal bone owing to approximately $60 \%$ of all tarsal fractures among adults [1]. Work related injury is mostly the main cause with fall from height being the reason in three fourth of the cases. The majority are young males [2] with an estimated male to female ratio of 2.4:1 [3]. The calcaneus fractures can be broadly divided into intra-articular and extra-articular. Extra-articular fractures were excluded from the study.

Patient will usually present with a history of axial loading and complains of heel pain and swelling and inability to bear weight. After the initial clinical assessment, radiologic imaging of the foot is the next diagnostic choice. Images include anteroposterior (AP), lateral and axial views. AP radiographs evaluate calcaneocuboid joint involvement, talonavicular subluxation and lateral wall widening. Lateral radiographs are done to calculate Bohler's angle. It is the angle defined by two intersecting lines: one drawn from anterior process of the calcaneus to the peak of the posterior articular surface and a second drawn from the peak of the posterior articular surface to the peak of the posterior tuberosity. The average angle is $25-40^{\circ}[4]$.

Management of calcaneus fractures have been a controversial among treating surgeons for a long time. Despite many classification 
schemes and advances in diagnostic and therapeutic tools, a true agreement on definitive treatment has not been reached [5]. Irregularity of the bone, complexity of the joint and the soft tissue envelope also makes these fractures a challenge. There is a school of thought that restoring Bohler's angle to near normal after fracture equates to better outcomes [6-7], but Randle et.al, for instance, mentioned in the meta-analysis that "there is a trend for surgically treated patients to have better outcomes; however, the strength of evidence for recommending operative treatment is weak" [8]

A high volume of cases with calcaneus fractures are encountered at our hospital primarily due to extensive construction work in the neighborhood. Due to the controversy in literature regarding treatment of the calcaneus fracture, we wished to determine whether operative treatment for intra-articular calcaneus fractures results in improvement of post-operative Bohler's angle and if the pre and postoperative measurements can be used as a determining and prognostic factor for clinical outcome. And finally, how has the treatment affected the patients' quality of life.

\section{Materials and Methods}

This retrospective study was conducted at the orthopedic department in Hamad General Hospital, Doha, Qatar which is a state of art tertiary care hospital and a level 1 trauma center. A waiver of consent was obtained for the purpose of study. Participants, wherever contacted, were informed and consented for participation. The study has been approved by the ethical committee at Medical Research Center, Hamad Medical Corporation (Study approval \# \#12202/12).

Patient files, medical records, radiologic images were reviewed by two independent observers. A total of 131 intra-articular calcaneus fractures from 117 patients in two years. Of these, 100 fractures from 96 patients were followed up. There were six cases of bilateral calcaneus fracture, of which four were operated bilaterally. For the purpose of radiologic analysis, number of fractures and not the number of patients were used. The mean age of these 96 patients was 35.6 years, with youngest patient of 19 years and the eldest 66 years. $92 \%$ of the fractures occurred in males.

Adults (>18 years) with closed intra-articular fractures that underwent open reduction internal fixation for the calcaneus fracture were included. Patients with extra articular fractures, open injuries, multiple injuries in the same foot, if they had closed reduction and fixation, if they were less than 18 years of age time of presentation, had known previous calcaneus pathology or if they were on any therapeutic treatment for bone diseases, as well as atraumatic or trivial injuries were excluded from the study.

The fractures were classified according to Bohler's angle. Arbitrary grading into three classes were done as defined by $\mathrm{C}$ Loucks et al. in 1999 [9]. Class A, if the initial measurement was at least $16^{\circ}$. Class $\mathrm{B}$, if the Bohler's angle was between $0-15^{\circ}$, and
Class $C$, if the initial Bohler angle was $<0^{\circ}$. There were 14 fractures in Class A, 52 of Class B, and 24 of Class C. 10 fractures were found where the initial Bohler's angle was $>25$ but underwent surgery due to surgeons' preference. These were not used while performing the analysis. Likewise, pre and postoperative angle of Gissane was also calculated but not used in further analysis. Sanders classification using the pre-operative CT films was also done but not utilized for the study.

All the patients that were included in the study underwent open reduction internal fixation using the lateral extensile approach as described by Benirschke and Sangeorzan [10] after the appearance of positive wrinkle test [11] (average 10 days). These patients were admitted during the whole duration after the injury, kept on leg elevation, ice packing and non-weight bearing protocol. As retrieved from the medical records, the operating surgeons were either senior orthopedic surgeons themselves or were physically present at the time of surgery. The average time for surgery is 97 minutes.

The pre and immediate postoperative radiographs were assessed by two independent examiners who were kept unaware of the details of the patient or the treating surgeons. The preoperative radiographs and CT scans were evaluated twice by two independent observers. Minimum follow up period for assessing clinical outcome was restricted to one year. Wherever possible, the clinical outcome was scored from the existing records and treating surgeons' notes. If unavailable, patients were telephonically followed up and assessed using the Foot Function Index [12] and American Orthopedic Foot and Ankle Society (AOFAS) Ankle Hind Foot Score [13] (subjective component only) by two independent observers.

Statistical Analysis: All statistical analysis was done using SPSS 20.0 (SPSS Inc. Chicago, IL). The inter-observer reliability was measured using the Fleisskappa [14] whereas the intra-observer reproducibility was measured using the Cohen's kappa [15] statistics.

\section{Result}

As reported earlier, these fractures were classified according to the Bohler's angle at the time of initial presentation. Total number of cases in each group were identified and recorded. There were total of 13 fractures with initial Bohler's angle between 16-25 (Class A), 51 fractures with Bohler's angle between $0-15^{\circ}$ (Class B), and 26 fractures with an initial Bohler's angle of less than $0^{\circ}$.

Inter-observer reliability was calculated using Fleiss-kappa and the kappa value was 0.6258 (95\% CI: 0.5902 to 0.7114 ) for preoperative and 0.6478 (95\% CI: 0.4487 to 0.6481 ) for postoperative measurements. The Cohen kappa coefficient for intra- observer reproducibility was 0.6141 (95\% CI: 0.4304 to 06578) for preoperative versus 0.6672 (95\% CI: 0.4753 to 0.8591 ) for postoperative measurement of Bohler's angle. 
Quantitative analysis was done using the paired t-test. The mean improvement in Bohler angle was found to be $13.14^{\circ}$ (p < $0.001)$ for Class A, $20.24^{\circ}(p<0.001)$ in the Class B group, whereas it was $44.75^{\circ}(\mathrm{p}<0.001)$ in class $\mathrm{C}$ fractures.

For the functional outcome, each group was scored (subjective component only) according to the AOFAS Ankle Hind-foot Score (Total score $=60$ ). The mean score for class $A, B$ and $C$ was found to be $46.38( \pm 9.86), 40.98( \pm 12.71), 26.23( \pm 14.17)$ respectively. Likewise, the mean Foot Function Index (FFI) was $25.66( \pm 3.39)$, $31.42( \pm 9.88), 57.63( \pm 11.40)$ for class A, B and C respectively Table [1-5], Figure 1.

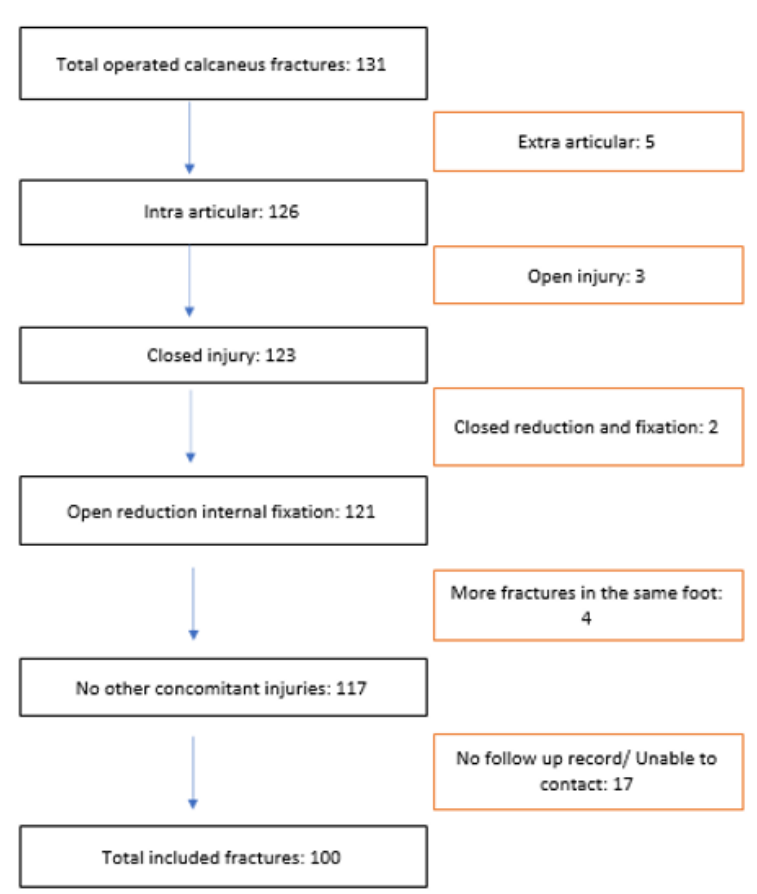

Figure 1: Schematic Flow chart: Calcaneus fractures included in the study.

Table 1: Percentage of fractures according to initial Bohler's angle.

\begin{tabular}{|c|c|c|}
\hline Class A & Class B & Class C \\
\hline $15 \%$ & $58 \%$ & $27 \%$ \\
\hline
\end{tabular}

Table 2: Inter-observer reliability (IOR).

\begin{tabular}{|c|c|c|c|}
\hline IOR & Results & $\mathbf{9 5 \%}$ CI & Interpretation \\
\hline Preoperative & 0.6258 & 0.5902 to 0.7114 & $\begin{array}{l}\text { Substantial } \\
\text { agreement }\end{array}$ \\
\hline Postoperative & 0.6478 & 0.4487 to 0.6481 & $\begin{array}{l}\text { Substantial } \\
\text { agreement }\end{array}$ \\
\hline
\end{tabular}

Table 3: Intra-observer reproducibility (IOR).

\begin{tabular}{|c|c|c|c|}
\hline IOR & Results & $\mathbf{9 5 \%}$ CI & Interpretation \\
\hline Preoperative & 0.6141 & 0.4304 to 06578 & $\begin{array}{l}\text { Substantial } \\
\text { agreement }\end{array}$ \\
\hline Postoperative & 0.6672 & 0.4753 to 0.8591 & $\begin{array}{c}\text { Substantial } \\
\text { agreement }\end{array}$ \\
\hline
\end{tabular}

Table 4: Improvement in Bohler's angle.

\begin{tabular}{|c|c|c|c|}
\hline \multirow{2}{*}{ Class } & Definition & $\begin{array}{c}\text { Mean } \uparrow \text { in Bohler } \\
\text { angle at post op day } \\
\mathbf{9 7}(\mathbf{6 3 - 1 2 7})\end{array}$ & p-value \\
\hline Class A & $($ Bohler angle 16-25 $)$ & 13.14 & $(\mathrm{p}<0.001)$ \\
\hline Class B & $($ Bohler angle $0-15)$ & 20.24 & $(\mathrm{p}<0.001)$ \\
\hline Class C & $($ Bohler angle $<0)$ & 44.75 & $(\mathrm{p}<0.001)$ \\
\hline
\end{tabular}

Table 5: Functional score.

\begin{tabular}{|c|c|c|c|c|}
\hline Class & AOFAS (Subjective only) & $\mathbf{9 5 \%}$ CI & FFI & $\mathbf{9 5 \%}$ CI \\
\hline Class A & 46.38 & \pm 9.86 & 25.66 & \pm 3.39 \\
\hline Class B & 40.98 & \pm 12.71 & 31.42 & \pm 9.88 \\
\hline Class C & 26.23 & \pm 14.17 & 57.63 & \pm 11.40 \\
\hline
\end{tabular}

\section{Discussion}

Treatment of calcaneus fractures is still a debatable issue among many orthopedic surgeons [16]. Many classification patterns [17] were proposed throughout the last century resulting in various treatment options being used for the same fractures with varying results.

One of the strengths of this work is the number of cases that were encountered with calcaneus fractures during the study period. Most studies are spanned over decades in order to capture enough cases for a favorable outcome. The study focusses on the functional and radiological outcomes of operative treatment on intra-articular treatment of calcaneus fractures based upon the traditional Bohler's angle. Despite the advent of latest radiological techniques including CT scan for defining calcaneal fractures, Bohler angle is still extensively used by orthopedic surgeons as a primary indicator for further treatment planning and as the main or only tool for postoperative assessment of cases. 
Both pre and postoperative Bohler's angle were included, and it was found that operative treatment with open reduction internal fixation of intra-articular calcaneal fractures is significantly associated with anatomical restoration of Bohler's angle. It has been demonstrated in various studies previously that Bohler's angle has prognostic relevance [18-20]. Our study reinforces the importance of using BA as a primary indicator in terms of decision making for intra-articular calcaneus fractures. There has been some controversy whether class A fractures should be treated surgically or non- operatively, but this study concludes that if the $\mathrm{BA}$ on presenting radiographs are $<25$, optimum results in terms of Bohler's angle can be achieved by operative treatment. Although, not included in the main study, but operative treatment was not found to be significantly associated in patients whose initial BA was $>25$ which further emphasize our theorem.

All cases that are included in the study were reviewed at one year to assess clinical outcome. AOFAS (subjective only) Hind-foot score and FFI scores were calculated to assess foot function in terms of pain, disability and activity restriction and they indicated good results. These subjective results accompanied by more objective findings advocate operative treatment for all intra-articular fractures with initial BA of $0-25^{\circ}$.

We recognize the fact that long term radiological evidence is missing, and the radiographs were not repeated to assess long term effect of operative treatment on BA. More than $80 \%$ of our cases are manual laborers and/ or construction workers. To them, key component of the treatment is pain-free walk and none-to-minimal restriction to activity. Although CT scan was done for most patients in our study, and the fractures were classified according to Sanders, but since our aim was focused to BA, they were not included in the further analyses. The study does not take into account results of non- operative calcaneus fractures at our settings as no equitable data could be found for comparative study. We are aware that a lot of patients that underwent operative treatment were lost during clinical follow-up, but it is due to the fact that majority of the patients who sustain calcaneus fracture are expats, and many leave the country after the trauma.

\section{Conclusion}

Preoperative measurement of BA is the key component in defining treatment plan for intra-articular calcaneus fractures.X-Ray, and not the CT-scan is still the method of choice for postoperative assessment of surgical fixation. Our study demonstrates that there is substantial reliability and reproducibility of Bohler's angle compared to Saunders classification system. Surgical fixation leads to restoration of Bohler's angle in all classes and good functional outcome in patients with initial Bohler's angle between $0-25^{\circ}$ and therefore indicated for this group of patients. We strongly advocate use of grading system of Bohler's angle that can substantially affect surgeons' decision regarding choice of treatment for intra-articular calcaneus fractures and can be predictably used as a prognostic indicator.

\section{Acknowledgement}

None.

\section{Conflict of Interest}

The authors and co-authors declare no conflict of interest.

\section{References}

1. Eiff MP, Hatch RL (2012) Fracture Management for Primary Care. Elsevier Saunders, Philadephia.

2. Sanders RW, Clare MP (2010) Calcaneous fractures, in Rockwood and Green's Fractures in Adults. Lippincott Williams \& Wilkins, Philadephia, USA, Pp. 2064.

3. Mitchell MJ (2009) The epidemiology of calcaneal fractures, 19(4): 197200.

4. Bohler L (1931) Diagnosis, pathology and treatment of fractures of the os calcis. J Bone Joint Surg Am: 75-89.

5. John D Maskill (2005) Calcaneus Fractures: A Review Article, Foot Ankle Clin N Am 10(3): 463-489.

6. Hildebrand KA (1996) Functional outcome measures after displaced intra- articular calcaneal fractures, J Bone Joint Surg Br 78(1): 119-123.

7. Kitaoka HB, EJ Schaap, EY Chao, KN An (1994) Displaced intra-articular fractures of the calcaneus treated non- operatively. Clinical results and analysis of motion and ground-reaction and temporal forces. J Bone Joint Surg Am 76(10): 1531-1540

8. Randle JA, HJ Kreder, D Stephen, J Williams, S Jaglal, R Hu (2000) Should calcaneal fractures be treated surgically: a meta- analysis. Clin Orthop Relate Res 377: 217-227.

9. Loucks C (1999) Bohler's angle: Correlation with outcome in displaced intra-articular calcaneal fractures. J Orthop Trauma 13(8): 554-558.

10. Benirschke SK, Sangeorzan BJ (1993) Extensive intraarticular fractures of the foot: surgical management of calcaneal fractures. Clin Orthop Relat Res: 128-134.

11. Sanders R (2000) Displaced intra-articular fractures of the calcaneus, J Bone Joint Surg Am 82(2): 225-250.

12. E Budiman-Mak, KJ Conrad, KE Roach (1991) The Foot Function Index: a measure of foot pain and disability. J Clin Epidemiol 44(6): 561-570.

13. Selene G Parekh (2009) Functional Assessment of Patients with Ankle Arthritis, in Arthritis and Arthroplasty: The Foot and Ankle, Philadelphia, Elsevier Inc.

14. Fleiss JL (1971) Measuring nominal scale agreement among many raters. Psychological Bulletin 76(5): 378-382.

15. Cohen J (1960) A coefficient of agreement for nominal scales. Educational and Psychological Measurement 1: 37-46.

16. Rowe CR, Sakellarides HT (1963) Fractures of the os calcis. JAMA: The Journal of the American Medical Association 184: 920-923.

17. Tim Schepers, Esther M M van Lieshout, Abida Z Ginai, Paul G H Mulder, Martin J Heetveld, Peter Patka (2009) Calcaneal fracture classification: a comparative study. J Foot Ankle Surg 48(2): 156-162.

18. Kundel K (1996) Calcaneal fractures: operative versus nonoperative treatment, J Trauma 41(5): 839-845.

19. Letournel E (1993) Open treatment of calcaneal fractures. Clin Orthop Relat Res 290: 60-67.

20. Loucks C (1999) Böhler's angle: correlation with outcome in displaced intraarticular calcaneal fractures, J Orthop Trauma 13(8): 554-558. 